\title{
The total virtual photoabsorption cross section, deeply virtual Compton scattering and vector-meson production
}

\author{
M. Kuroda ${ }^{1,2}$, D. Schildknecht ${ }^{2}$ \\ 1 Institute of Physics, Meiji Gakuin University, Yokohama 244, Japan \\ 2 Fakultät für Physik, Universität Bielefeld, 33501 Bielefeld, Germany
}

Eur. Phys. J. C 37, 205-222 (2004) - DOI: 10.1140/epjc/s2004-01986-x

Published online: 2 September 2004

Erratum published online: 2 November 2005 - (c) Springer-Verlag / Società Italiana di Fisica 2005

1. The left-hand side of (2.25) should read

$$
\sigma_{(q \bar{q})_{T, L}^{J=1} p}\left(r_{\perp}^{\prime 2}, W^{2}\right) .
$$

2. In the third line after (2.34), (2.21) should read (2.24).

3 . We have found a coding error in the computer program, which affects some of the cross sections of the massive vector-meson production. The sentences between (4.42) and (4.43) starting with "For $J / \Psi$ production, ......" should read:

For $J / \Psi$ production, for $Q^{2}+M_{J / \Psi}^{2} \geq 25 \mathrm{GeV}^{2}$, the approximation (4.37) overestimates the result from (4.39) by about $15 \%$. For the case of $\Upsilon$ production, for $Q^{2} \cong 0$, for later reference we note that the approximate result from (4.37) is about 30\% larger than the result of the exact evaluation.

Table 1 changes as follows:

\begin{tabular}{lllllll}
\hline$V$ & $R^{\left(\rho^{0}\right)} / R^{(V)}$ & $M_{V}(\mathrm{GeV})$ & $\Delta M_{V}^{2}\left(\mathrm{GeV}^{2}\right)$ & $m_{q}(\mathrm{GeV})$ & $E_{\text {appr. }}^{(V)}$ & $E^{(V)}$ \\
\hline$\rho^{0}$ & 1 & 0.77 & 1.0 & 0 & - & - \\
$J / \psi$ & $\frac{9}{8}$ & 3.096 & 3.0 & 1.5 & 1.34 & 1.32 \\
$\Upsilon$ & $\frac{9}{2}$ & 9.460 & 11.0 & 4.6 & 3.29 & 2.99 \\
\hline
\end{tabular}

The last two sentences in Sect. 4.2 should read: The deviation from the results from (4.43) for the $J / \Psi$ is small, since the excesses of the approximation (4.10) for the $\rho^{0}$ meson and of the approximation (4.37) for the $J / \Psi$ meson with respect to the exact result cancel. The larger deviation in the case of the $\Upsilon$ is largely due to the above mentioned $30 \%$ deviation of the approximation from the exact result.
4. Correspondingly, the right-hand sides of (4.50) and (4.51) change as

$$
\begin{gathered}
\frac{9}{8} \sigma^{(J / \Psi)} / \sigma^{\left(\rho^{0}\right)}=1.28, \\
E^{(\Upsilon)}=\frac{9}{2} \sigma^{(\Upsilon)} / \sigma^{\left(\rho^{0}\right)}=2.99,
\end{gathered}
$$

respectively.

5. In Sect. 4.4, in the second paragraph,

$$
\left(1 / Q^{2}\right)^{6} \alpha_{s}\left(Q^{2}\right) x g\left(x, Q^{2}\right)
$$

and

$$
\left(1 / Q^{6}\right) \sigma^{(\infty)} \Lambda^{2}\left(W^{2}\right)
$$

should read

$$
\begin{gathered}
\left(1 / Q^{2}\right)^{6}\left[\alpha_{s}\left(Q^{2}\right) x g\left(x, Q^{2}\right)\right]^{2} \\
\left.\left(1 / Q^{6}\right)\left[\sigma^{(\infty)}\right) \Lambda^{2}\left(W^{2}\right)\right]^{2}
\end{gathered}
$$

\section{and}

respectively. 